\title{
Modeling Neural Mechanisms of Vertebrate Habituation: Locus Specificity and Pattern Discrimination
}

\author{
DELIANG WANG \\ dwang@cis.ohio-state.edu. \\ Laboratory for AI Research, Department of Computer and Information Science, and Center for Cognitive Science, \\ The Ohio State University, Columbus, OH 43210, USA
}

Received August 5, 1993; Revised May 4, 1994; Accepted (in revised form) May 4, 1994.

Action Editor: S. Shamma

\begin{abstract}
A critical problem in neurobiology is to explain how the central nervous system coordinates pattern discrimination and locus specificity in learning. This problem is investigated in anuran amphibians who demonstrate both locus specificity and pattern discrimination in visual habituation. A neural mechanism is proposed whereby neural circuitry for pattern discrimination is shared by a spatial memory system. Such learning processes are argued to occur in the medial pallium (MP), the anuran's homolog of mammalian hippocampus. Necessary mapping from the shared network to spatial memory is set up by a mechanism that forms topographical connections, with desired orientation determined by activity gradient in presynaptic and postsynaptic layers. The model of MP is tested on both locus and stimulus specific habituation, which involve short-term as well as long-term synaptic plasticity. Successful modeling yields a set of predictions concerning MP organization and learning properties.
\end{abstract}

\section{Introduction}

Orienting and prey-catching behavior in amphibians can be released by an appropriate moving visual stimulus. This prey-catching behavior is subject to various kinds of modulation. In particular, the amplitude of the behavior is gradually reduced if the stimulus is repeatedly presented. Interestingly enough, visual habituation in toads and frogs exhibits:

(1) Locus specificity. After habituation of an orienting response to a certain stimulus applied at a given location, the animal responds again to the same stimulus applied at a different visual location (Eikmanns, 1955; Ewert and Ingle, 1971). As shown in Fig. 1, habituation to a stimulus at a particular visual location was obtained by a series of habituation trials with an inter-trial pause of $1 \mathrm{~min}$ for recovery. Within each trial, a prey dummy was continuously presented until the animal's response diminished. A habituation series was terminated if there was no response at all during a single trial. After a habituation series was completed at a specific visual location, the stimulus was then presented at a different location to start a new habituation series. After habituation at a specific location, the toad's response was facilitated to the same stimulus applied at a location nasal to the habituation spot, and the habituation series was prolonged (Fig. 1A); whereas the animal's response was inhibited at a location temporal to the habituation spot, and the habituation series was shortened (Fig. 1B). Clearly locus specificity in anurans involves intraocular interactions.

(2) Stimulus specificity. Another stimulus given at the same locus may "dishabituate" the animal, yielding a response at a site habituated by a previous stimulus. Only certain stimuli can dishabituate a previously habituated response. Ewert and Kehl (1978) showed that this dishabituation forms a hierarchy (Fig. 2A), where a stimulus can dishabituate the habituated responses of another stimulus if the latter is lower in the hierarchy or on the same level but to the right. This basic finding was further confirmed and extended by a recent behavioral study conducted by Wang and Ewert (1992), who showed this dishabituation does not remove the prior habituation. The dishabituation hierarchy demonstrates that the animal has the capacity of discriminating similar visual patterns. This capacity distinguishes anuran habituation from that of invertebrates, like Aplysia (Kandel, 1976), where habituation does not seem to be independent of specific patterns used. 

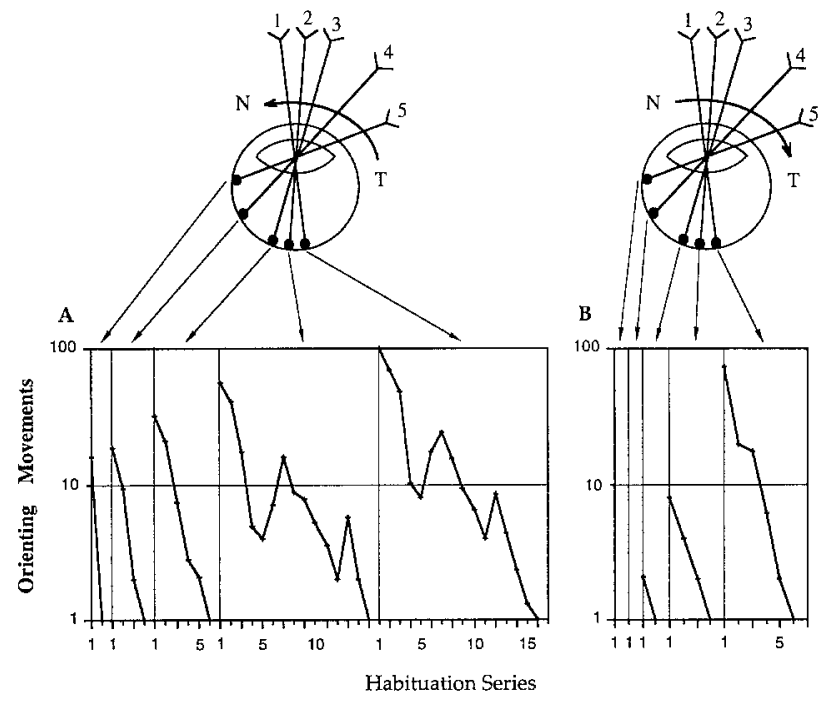

Fig. 1. Locus specific habituation of prey-catching orienting response in toads. The prey dummy was a $4^{\circ} \times 20^{\circ}$ stripe. Five tested visual locations, labeled as $1,2,3,4,5$, all lay to the right side of the animal, and corresponded to $30^{\circ}, 45^{\circ}, 60^{\circ}, 90^{\circ}$, and $120^{\circ}$ angles, respectively. A Habituation series conducted from temporal to nasal locations in the order of 5-4-3-2-1. B Habituation series conducted from nasal to temporal locations in the order of 1-2-3-4-5. $N$ denotes nasal and $T$ temporal. Abscissa: habituation trial number. Ordinate: cumulated number of orienting turns for each trial plotted on the log-scale. Within each series, time runs from left to right (redrawn from Ewert and Ingle, 1971).

Besides locus and stimulus specificity, the orienting behavior in toads and frogs also exhibits other typical properties of habituation as summarized by Thompson and Spencer (1966). These include the exponential decrease of response intensity, spontaneous recovery, and both short-term and long-term habituation that last for different time periods (for a review see Ewert, 1984; Ewert et al., 1992).

In a model of toad's visual habituation, Lara and Arbib (1985) have attempted to model locus specificity by lateral interaction. Probably due to the lack of a model of long-term habituation, they were not able to simulate habituation series, and they provided only an incomplete comparison between their model outputs and the experimental data, which do not appear in good accord.

We have previously carried out a series of computational studies searching for the visual pathway and neural mechanism that underlie pattern discrimination and learning in anuran amphibians. In Wang and Arbib (1991), we demonstrated that worm pattern discrimination can be achieved in the anterior thalamus (AT), a group of AT cells responding with larger intensity to stimuli higher in the dishabituation hierarchy. In Wang and Arbib (1992), concluding from experimental evidence from anatomical, physiological, and lesion studies, we suggest that the medial pallium (MP), the homolog of the mammalian hippocampus ("primordium hippocampi" by Herrick, 1933), is the brain region where habituation and other forms of learning occur. Furthermore, we modeled one MP column, as shown in Fig. 2B, which consists of five types of neurons, three of which (MP1, MP2 and MP3) have been identified physiologically (Finkenstädt, 1989) and the other two (P1 and P2) were hypothesized. Using the MP column model, we successfully simulated detailed dishabituation relationships leading to the dishabituation hierarchy. In a recent study, we addressed both short-term and long-term habituation of preycatching behavior by two interacting differential equations describing synaptic plasticity underlying habituation (Wang, 1993). Computer simulations show that the parsimonious description of synaptic plasticity can explain long-term habituation data over a period of several weeks.

The present study builds on the previous work, and the earlier models of the visual pathway below telencephalon serve to provide front-end input to present modeling. In particular, we investigate how locus specificity and the neural circuitry for pattern discrimination can be coordinated, given that similar pattern discrimination occurs at all visual locations. A straightforward idea would be to duplicate the discrimination network for every discernible locus. Two major prob- 
A

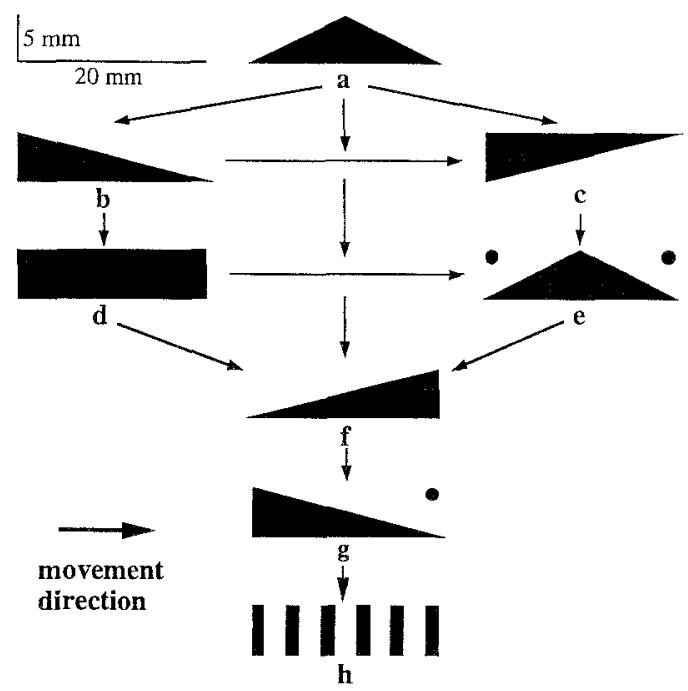

B

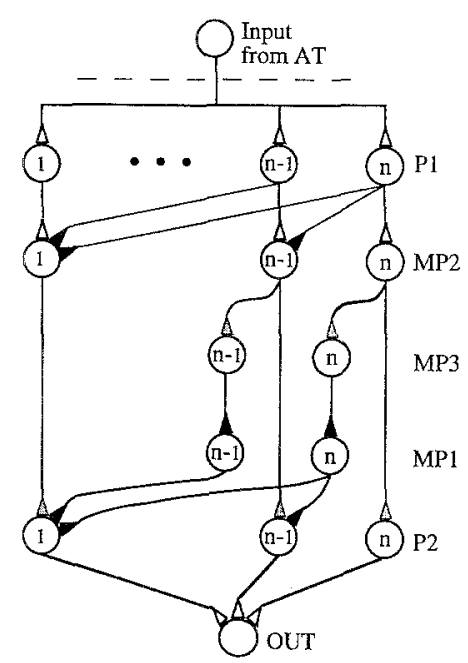

Fig. 2. A Dishabituation hierarchy for worm stimuli used in stimulus-specific habituation. One stimulus can dishabituate all the stimuli below it. On the same level the left one can slightly dishabituate the right one (redrawn from Ewert and Kehl, 1978). B Single MP column model. Each cell type is represented by a layer of $n$ cells. Empty triangles denote excitatory, black ones inhibitory, and filled ones habituation synapses (redrawn from Wang and Arbib, 1992).

lems with this idea are its hardware inefficiency due to a large amount of duplication, and the potential for independent hardware to develop inhomogeneities due to idiosyncratic neural plasticity within a locus, leading to an undesired asymmetry for different visual loci. It appears that different loci sharing the same discrimination circuitry is a superior idea. We study how this idea might be realized in anuran amphibians, leading to a neural model of MP organization. Since locus specificity involves interactions between different loci, simply modeling each locus independently does not work. This issue will be addressed when modeling the structure of MP. As will be clear in Sect. 4, longterm synaptic plasticity must be modeled in order to simulate habituation series. With a model of synaptic plasticity that exhibit both short- and long-term properties, we can now quantitatively simulate habituation series performed at each visual locus, and provide a full comparison with the quantitative experimental data of locus specificity.

\section{Neural Organization of the Medial Pallium}

The medial pallium in anuran amphibians is a large neural structure of the "nonolfactory" telencephalon, surrounding the telencephalic ventricle. It is the major integrative center for all modalities of sensory information. Anatomical studies reveal at least four morphologically classified cell types, and indicate that this region is organized in an orientation vertical to the telencephalic ventricle (Hoffman, 1963; Kicliter and Ebbesson, 1976; Northcutt and Kicliter, 1980; Neary, 1990). Neuronal projections exist in directions both parallel and perpendicular to the pial surface. All anatomical evidence leads us to suggest that the medial pallium processes information by means of functional units of vertical columns. One functional advantage of such columnar organization is that each column can naturally store a set of patterns independently, consistent with locus specificity of learning. The present work is only concerned with the MP role in visual processing.

Based on connectional and neuroethological studies, Ewert (1987) suggested two neural loops to explain prey-catching behavior and its modulation in amphibians. Loop(1) mediates prey-catching behavior. It involves the tectum which is the major recipient of retinal efferents, the lateral anterior thalamic nucleus, the striatum, and the thalamic-pretectal region. Loop(2) starts with the retina and the tectum which send axons to AT and from there to MP, which then has a descending projection to the preoptic region $(\mathrm{PO})$ and the hypothalamus (HYP), which in turn send efferents to the tectum (retina $\rightarrow$ tectum $\rightarrow A T \rightarrow M P \rightarrow$ $P O / H Y P \rightarrow$ tectum). Loop(2) is supposed to modulate prey-catching behavior initiated in the tectum. All data obtained to date strongly suggest that MP is the neural structure where various types of learning occur. For stimulus specific habituation, MP modulates 


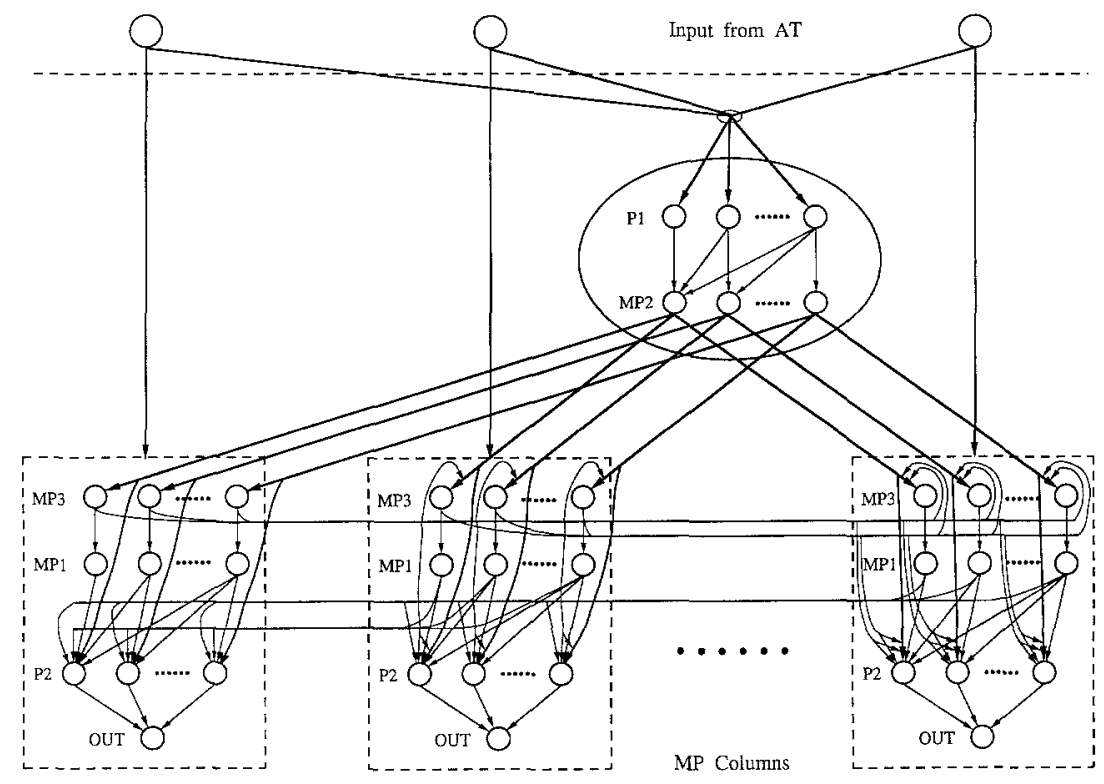

Fig. 3. Diagram of the MP model. The P1 layer and the MP2 layer form an independent neural network between AT and MP columns which are denoted by dashed line boxes. Arrowheads represent synapses, and arrowheads projecting on connection lines or other arrowheads represent presynaptic synapses. See Fig. 2B for detailed types of synapses.

prey-catching behavior via the PO/HYP pathway, inhibiting tectal activities (Ewert, 1987; Wang and Arbib, 1992). Physiological recordings have identified three types of visually sensitive neurons in MP which exhibit spontaneous firing activities (Finkenstädt, 1989). MP1 neurons strongly increase, MP3 neurons decrease, and MP2 neurons do not alter their discharge rates in response to repetitive stimulation by a visual moving object.

Functionally, the MP column model (Fig. 2B) previously proposed has two main parts: Layer P1 and MP2, based on the cumulative shrinking mechanism, convert AT firing activity into a spatial distribution of neuronal activities in an MP2 cell group. Cumulative shrinking reduces the activity along one direction of a neural layer based on the cumulative activity from the other direction and normalizes the entire layer. The rest of the column builds the memory for visual patterns, and implements interactions between the memories of different worm-like stimuli underlying the dishabituation hierarchy. To exhibit locus specificity, a separate memory for each discernible visual locus must be present, and thus the second part of the column cannot be shared by different loci. However, neural computation of the first part may be shared by different loci, since it is transient. Because anurans can single out one visual target when facing multiple objects si- multaneously, possibly on the basis on circuitry in the tectum (Didday, 1976), there is no interference due to simultaneous use. To simplify discussion, we assume that each AT efferent to MP represents one distinct visual location. This assumption is supported by the fact that AT is organized retinotopically (Montgomery and Fite, 1989).

As shown in Fig. 3, we model the medial pallium as an array of columns with a common network consisting of P1 and MP2 layers (Fig. 2B), called the conversion network. The conversion network receives inputs from all AT neurons that cover the entire visual field. Each MP column consists of the MP3, MP1, and P2 layers, as well as the sole output cell OUT. Each column receives a gating input from a corresponding AT neuron, signaling a stimulus at a specific visual locus. In addition, the MP3 and P2 layers receive in register one-to-one inputs from the MP2 layer of the conversion network. Therefore, as far as one visual locus is concerned, the MP column plus the conversion network forms the same circuitry as the previous single column model of Fig. 2B.

In the model, as well as in the physiological recordings, MP3 cells decrease and MP1 cells increase their spontaneous activities after habituation to a visual stimulus. To address intercolumnar interactions, we hypothesize that the MP3 cells of an MP column uni- 
laterally inhibit the $\mathrm{P} 2$ layers of columns to its right direction, and the MP1 cells unilaterally inhibit the P2 layers of columns to its left. To map the model's topography to the visual field, we assume that left columns of Fig. 3 correspond to the temporal side of the visual field and right columns to the nasal side. Qualitatively, after habituation of a specific MP column, columns to its right (the nasal direction) are facilitated in response to a visual stimulus at their corresponding visual locations due to reduced MP3 inhibition from the left direction, whereas columns to its left (the temporal direction) are inhibited due to increased MP1 inhibition from the right direction. Additionally, the MP3 cells of a column also unilaterally project to habituation synapses from layer MP2 to layer MP3 via presynaptic connections to influence the time course of habituation. These habituation synapses are subject to habituation based on presynaptic stimulation. To reduce intercolumnar connections, MP3 and MP1 projections can be integrated by single interneurons before leaving a column, as indicated in the figure as a single line. The quantitative description is presented in Sect. 4. We include only an array of columns instead of an entire two dimensional surface because there are no data available regarding intercolumnar interactions along the vertical direction. At present, one can imagine that the entire MP surface is an isolated row of identical MP arrays.

It is rather strict to require that the MP2 layer of the conversion network project in register to the MP3 and P2 layers of each MP column. A question naturally arises: how are these topographical connections formed during ontogenesis from one layer of neurons uniformly to some layers of neuronal columns which themselves are organized topographically? In the next section, we propose a theory about how these topographical maps might be formed in early development.

\section{Formation of Topographical Projections}

It is reasonable to assume that before the formation of MP columns, the MP2 layer projects uniformly onto the entire surface of MP3 and P2 layers, and each ascending AT fiber innervates a focal area of about the same size in the MP2 array. Figure 4A illustrates the situation with only MP3 since it is similar with P2. Each AT fiber activates its focal area as long as some visual stimulus falls in its receptive field. Since different AT fibers are generally activated at different times in early visual experience, the entire arrays of MP3 and P2 may be broken into independent regions, forming
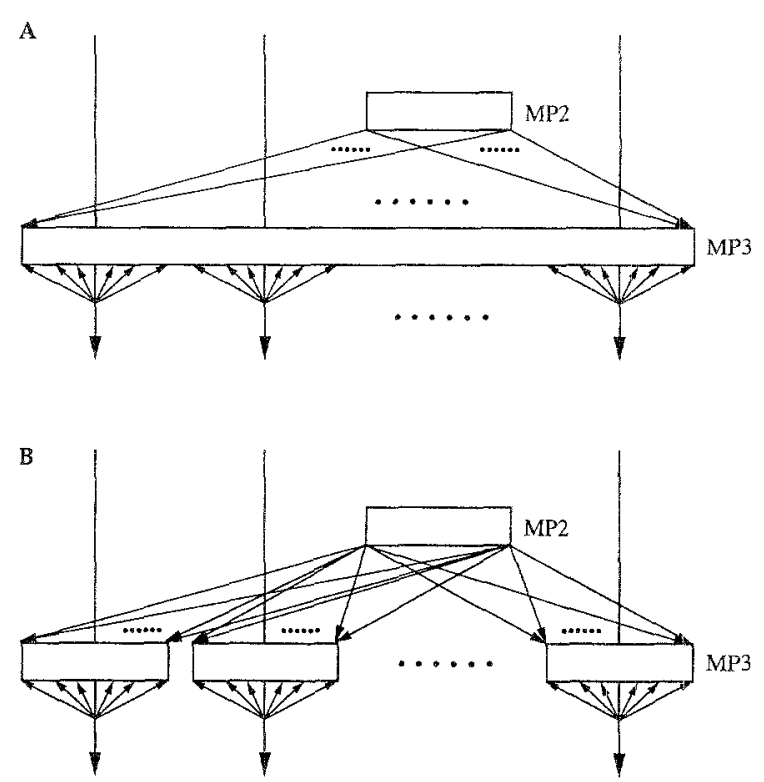

Fig. 4. Formation of MP columns. A An early stage connection diagram from MP2 to MP3. B A later stage diagram when the MP3 layer is broken into subregions. Vertical lines represent AT fibers.

MP columns (Fig. 4B). This process can be explained by a neural coactivation rule, which binds cells within each column together due to coactivation by the same AT fiber.

The question remains how to form a topographical mapping from the MP2 layer to an MP column from the initial all-to-all correspondence of Fig. 4B? The general question of forming topographical (e.g., retinotectal) maps has been studied by a number of modelers. Among others are the arrow model of Hope et al.(1976), the neural activation model of Willshaw and von der Malsburg (1976) and its extension by Amari (1980), the chemical marker model of von der Malsburg and Willshaw (1977), a hybrid of the arrow model and the chemical marker model of Overton and Arbib (1982). More recently, Kohonen's algorithm of self-organizing feature maps (1982) has been applied to form the socalled cortical maps which include both position and orientation (Durbin and Mitchison, 1990; Obermayer et al., 1990). Among them, the model of Willshaw and von der Malsburg and Amari's extension are the only models that are directly realizable by neural operations. Both the arrow model and Kohonen's algorithm are algorithms, yet to be implemented neurally. The chemical marker model, on the other hand, involves a chemical process, like chemoaffinity and diffusion.

We seek a neurally realizable model that can account for topographical mapping from the MP2 layer 
to each MP column. In addition, the model requires that the topographical mapping from MP2 to all MP columns have a single global orientation, say, the left end of layer MP2 to the left end of all MP columns as drawn in Fig. 4. Several ways have been suggested to determine the orientation of topography. Willshaw and von der Malsburg (1976) suggested to have one nucleation region with some initially strong connections that convey orientation. It was later suggested that the initial retinotectal connections provide a rough bias towards the desired orientation (von der Malsburg and Willshaw, 1977; Amari, 1980). Both suggestions rely on initial synaptic connections to determine final orientation. It should be clear that ultimate information about orientation must be provided genetically. Although these suggestions seem reasonable for mapping one layer to another, it is unnatural to adopt them in the present situation, because one MP2 layer needs to symmetrically map on multiple columns. The scheme of the initial connection bias would require that connections from the MP2 layer to all MP columns have the same bias. But as illustrated in Fig. 4, the projections from layer MP2 to the entire surface of MP3 are likely to be homogeneous at the beginning, so the same bias would be unnatural to develop later within each column.

In the following model, we hypothesize that global orientation is determined by neural activities in both presynaptic and postsynaptic layers, not by synaptic connections. More specifically, there is a slight gradient in firing activities of the MP2 layer, consistent with the model of layer MP2 (Wang and Arbib, 1992). In addition, there is a slight gradient in MP3 layer of each MP column, possibly due to innervation from an AT fiber. As demonstrated below, if the direction of the activity gradient of a postsynaptic layer is the same for all MP columns, the final orientation for all maps will be the same. This condition can be assured by a simple assumption that all AT fibers have the same innervation pattern. Due to the activity gradient, the presynaptic end with stronger neuronal activity has the best chance to first activate the postsynaptic end with stronger neuronal activity. A topographical map can thus be first developed between the stronger ends of both presynaptic and postsynaptic layers, and the map can then extend to the rest of the connections until a full topographical map is established.

Let $W_{i j}$ be the synaptic weight from cell $j$ in an array of $M$ presynaptic cells to cell $i$ in an array of $N$ postsynaptic cells. Let $m_{r}(j), N_{r}(j)$ be the membrane potential and the output of cell $j$ in the presynaptic layer respectively, and $m_{s}(i), N_{s}(i)$ those of $i$ in the postsynaptic layer. Then,

$$
\begin{aligned}
\frac{d m_{s}(i, t)}{d t}= & -\lambda m_{s}(i, t)+\sum_{j} W_{i j} N_{r}(j, t) \\
& +\sum_{k} S(k-i) N_{s}(k, t)
\end{aligned}
$$

where $t$ denotes time, and $\lambda$ is the decay constant. The second summation represents lateral connections within the postsynaptic layer, the even function $S(x)$ representing the weights of lateral connections. $S(x)$ is positive for small $|x|$ and negative for large $|x|$, exhibiting the shape of a Mexican hat. The output of cell $i$

$$
N_{s}(i, t)= \begin{cases}m_{s}(i, t)-\theta_{s}(i) & \text { if } m_{s}(i, t)>\theta_{s}(i) \\ 0 & \text { otherwise }\end{cases}
$$

where $\theta_{s}(i)$ is the threshold of cell $i$. To ensure that initial synaptic weight $W_{i j}$ 's have no particular preference, they are randomly chosen from a small range of positive values. The following procedures are taken for each cycle, during which the postsynaptic neurons are stimulated by a given set of presynaptic neurons.

(1) A small segment of $c$ presynaptic cells is randomly chosen to activate from the presynaptic layer. That is, those $m_{r}(j)$ 's are set to 1 , the rest are set to 0 . $N_{r}(j)$ is defined as

$$
\begin{aligned}
& N_{r}(j, t) \\
& = \begin{cases}m_{r}(j, t)-\theta_{r}(j) & \text { if } m_{r}(j, t)>\theta_{r}(j) \\
0 & \text { otherwise }\end{cases}
\end{aligned}
$$

where $\theta_{r}(j)$ is the threshold of cell $j$. The values of $\theta_{r}(j)$ and $\theta_{s}(i)$ that monotonically change with $j$ or $i$ determine the activity gradients of the presynaptic and postsynaptic layers respectively.

Small segments of presynaptic cells are chosen to correspond to the natural stimuli present in the structured environment where the animal lives in. In general, the visual environment for toads can be viewed as composed of many stripes, such as tree leaves, grass stems, and small worms and insects (Ewert, 1984). Cell layers with systematic threshold changes have been identified in the oculomotor and abducens nuclei (Schiller, 1970) and in the auditory periphery (Liberman, 1978). This property has been used in a neural network model by Grossberg and his colleagues to generate the position-threshold-slope shift map that converts different input intensities to different positions in an 
array of neurons (Grossberg and Kuperstein, 1986, pp. 160-167; Grossberg and Schmujuk, 1989).

(2) Each of the $N$ postsynaptic cells updates its membrane potential and generates an output according to (1) and (2) until the absolute change in membrane potential is less than $3 \%$ (close to equilibrium), or the number of updates exceeds 2000.

(3) Synaptic weight $W_{i j}$ is changed according to a Hebbian rule of plasticity (Hebb, 1949)

$$
\Delta W_{i j}=\eta N_{s}(i, t) N_{r}(j, t)
$$

where $\eta$ controls the speed of synaptic modification.

(4) The synaptic weights are then normalized so as to keep the mean strength associated with each postsynaptic and presynaptic cell at a constant value (see von der Malsburg, 1973; Willshaw and von der Malsburg, 1976),

$$
\begin{aligned}
& \sum_{j=1}^{M} W_{i j}=M \quad \text { for } i=1,2, \ldots, N \\
& \sum_{i=1}^{N} W_{i j}=N \quad \text { for } j=1,2, \ldots, M
\end{aligned}
$$

The above model was simulated between two layers of 20 cells each $(M=N=20)$. The activity gradient in the presynaptic layer was defined by $\theta_{r}(j)=$ $0.4 j / M$; and the postsynaptic gradient $\theta_{s}(i)=3.64+$ $0.8 i / N$. The initial values of the weight matrix $W_{i j}$ 's were uniformly chosen from the interval $[1.0,1.02]$. The strengths of lateral connections within the postsynaptic layer were set to the following values

$$
\begin{array}{lllcc}
x & 1 & 2 & 3 & 4 \\
S(x) & 0.2 & 0.1 & -0.2 & -0.4
\end{array}
$$

These lateral connection strengths were chosen according to the difference of Gaussian distributions (DOG). We found that the detailed values do not matter much.

Figure 5 shows the simulation result. Figure $5 \mathrm{~A}$ shows an early stage of developing a topographical map after 150 cycles. It illustrates that the map started from the projections between the presynaptic end with stronger activity (smaller thresholds) and the postsynaptic end also with stronger activity. Figure 5B shows the final stable and orderly map produced from the initial all-to-all mapping after 3000 cycles.

The simulation demonstrated the one-dimensional case, consistent with the MP model. The principle can be similarly applied to higher dimensional cases. The same process is assumed to take place from the
A

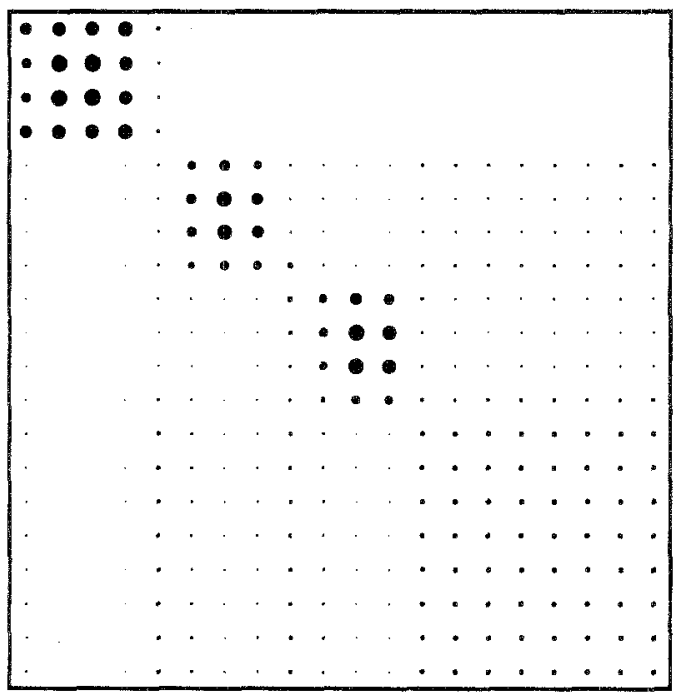

B

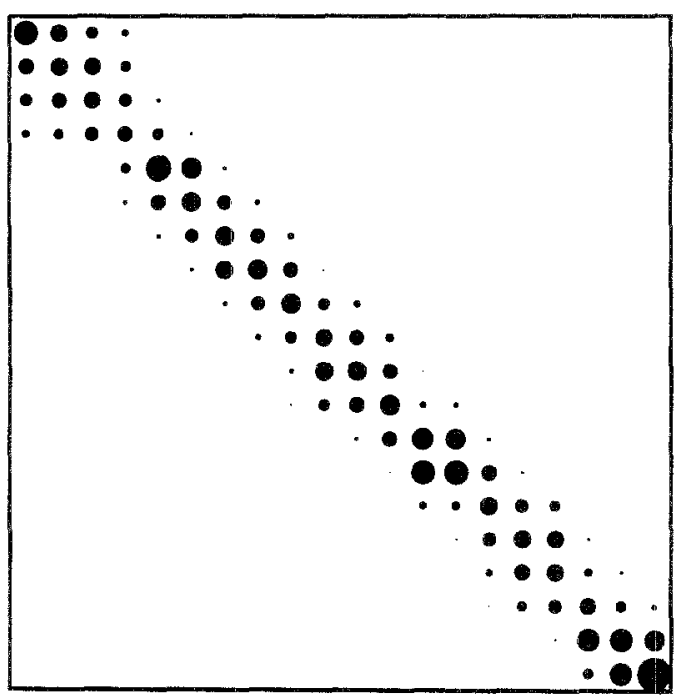

Fig. 5. Formation of topographical mapping. The connection pattern between two layers is represented by a connection matrix. A An early connection pattern after 150 cycles of iterations. B A map produced after 3000 cycles. The parameters $\lambda=0.5, c=2, \eta=0.2$, and $\Delta t=0.01$.

MP2 layer to the MP3 layer and the P2 layer in every MP column (Fig. 4B). In the MP model, topographical maps thus formed are further simplified to one-to-one maps, as shown in Fig. 3. In a sense, the solution provided here can be viewed as building submaps (Fig. 4B) within a grand map (Fig. 4A). Because of this requirement, previously proposed schemes for forming the desired orientation do not seem to fit the situation. More discussions of this topic are provided in Sec. 5 . 


\section{Formal Description and Computer Simulation}

Having discussed ontogenesis of the MP model, we now describe intercolumnar interactions. As shown in Fig. 3, the P2 layer of a column receives MP3 inhibition from left columns and MP1 inhibition from right columns. Assume that columns from left to right (Fig. 3) are labeled by small to large numbers. Integrating inhibition, the membrane potential and the output of the $j$ th $\mathrm{P} 2$ cell of column $i$,

$$
\begin{aligned}
& \frac{d m_{p 2}(i, j, t)}{d t}= \\
& -A_{p 2}(i) m_{p 2}(i, j, t)+y(i, j, t)\left(N_{m p 2}(j, t)-h_{1}\right) \\
& -B_{p^{2}} \sum_{k>j}\left(N_{m p 1}(i, k, t)-C_{m p 1}\right)-m_{p 2}(i, j, t) \\
& \times\left[\sum_{k<i} L_{k} \sum_{j^{\prime}}\left(N_{m p 3}\left(k, j^{\prime}, t\right)-C_{m p 3}\right)\right. \\
& \left.+\sum_{k>i} R_{k} \sum_{j^{\prime}}\left(N_{m p 1}\left(k, j^{\prime}, t\right)-C_{m p 1}\right)\right] \quad(6) \\
& N_{p 2}(i, j, t)=\operatorname{Max}\left(m_{p 2}(i, j, t), 0\right)
\end{aligned}
$$

where $A_{p 2}(i)$ is a relaxation parameter, and $y(i, j, t)$ is the modifiable weight of the projection from cell MP2 $j$ of the same column, to be described below. $N_{m p 2}(j, t)$ represents the output of the $j$ th MP2 cell at time $t$, and $N_{m p 3}(k, j, t)$ and $N_{m p 1}(k, j, t)$ represent the outputs of $j$ th MP3 and MP1 cells of column $k$ respectively. The formal description of these variables was given in Wang and Arbib (1992), and is provided in the Appendix for completeness. The term $\left(N_{m p 2}(j, t)-h_{1}\right)$ detects external stimulation from the MP2 layer, which is above the level of spontaneous firing $h_{1} . B_{p 2}$ is the strength of intracolumnar left inhibition (Fig. 2B), and $C_{m p 1}$ and $C_{m p 3}$ are the resting activities of an MP1 cell and an MP3 cell respectively before habituation, and they are given in the Appendix. The last factor of the above formula describes intercolumnar shunting inhibition (Grossberg, 1976). Multiplicative shunting inhibition is suggested from the data in Fig. 1 plotted on a log-scale (see the right two curves of Fig. 1A in particular). $L_{k}$ is the weight of overall MP3 inhibition from a right column $k$, and $R_{k}$ is the weight of overall MP1 inhibition from a left column $k$.

The animal's prey-catching response to the stimulus is not homogeneous across the visual field. The response is clearly stronger when the stimulus is more towards the nasal direction, when one compares the remarkable difference between the first habituation series to the stimulus at the $120^{\circ}$ angle (the leftmost curve of Fig. 1A) and the first habituation series to the stimulus at the $30^{\circ}$ angle (the rightmost curve of Fig. 1B). This inhomogeneity could be caused at various visual centers along the pathway from retina to MP, and for simplicity this is modeled in part by choosing different $A_{p 2}$ 's for different MP columns. After analyzing the data in Fig. 1 and numerous simulation trials, we found that the exponential relationship between the value of $A_{p 2}$ and the position of an MP column best suits the data. The same analysis applies to choosing parameter values $L_{k}$ 's and $R_{k}$ 's. Finally,

$$
\begin{aligned}
A_{p 2}(i) & =2^{\left(D_{i}-30\right) / 20} ; \quad R_{i}=2^{\left(120-D_{i}\right) / 85} ; \\
L_{i} & =2^{\left(D^{i}-30\right) / 10}
\end{aligned}
$$

where $D_{i}$ represents the visual degree that column $i$ corresponds to.

Modification of the synaptic weight $y(i, j, t)$ is described as (cf. Wang and Arbib, 1992; Wang, 1993)

$$
\begin{aligned}
& \tau \frac{d y(i, j, t)}{d t}=\alpha z(i, j, t)\left(y_{0}-y(i, j, t)\right) \\
& \quad-\beta y(i, j, t)\left(N_{m p 2}(j, t)-h_{1}\right) \\
& \frac{d z(i, j, t)}{d t} \\
& \left.=G(i) z(i, j, t)(z(i, j, t)-1) N_{m p 2}(j, t)-h_{1}\right) \\
& \quad\left(1+\sum_{k<i} L_{k} \sum_{j^{\prime}}\left(N_{m p 3}\left(k, j^{\prime}, t\right)-C_{m p 3}\right)\right)
\end{aligned}
$$

where $\tau$ is the time constant for controlling the rate of habituation, or reduction of $y(i, j, t)$ with the initial value $y(i, j, 0)=y_{0}$. The first factor in (9) regulates recovery towards the initial value. The product $\alpha z(i, j, t)$ has an activity dependent control on the rate of forgetting. The second factor causes habituation by the input from layer MP2, parameter $\beta$ controlling the speed of habituation. Equation (10), which essentially exhibits an inverse $S$-shaped curve with presynaptic input from the MP2 cell (ignore the intercolumnar influence of the last term for the time being), is used to control the speed of forgetting of habituation. The $S$ shaped curve of $z(i, j, t)$ has two stages of decrease: the first fast-decreasing stage and the second slowdecreasing one. The transition speed from the first stage to the second one is controlled by parameter $G(i)$. The larger is $G(i)$, the quicker is the transition. The overall speed of the decrease of $z(i, j, t)$, however, is controlled by the initial value $z(i, j, 0)$, and the larger $z(i, j, 0)$ the slower is the speed of decrease. The two phases are used to model two stages of habituation: 


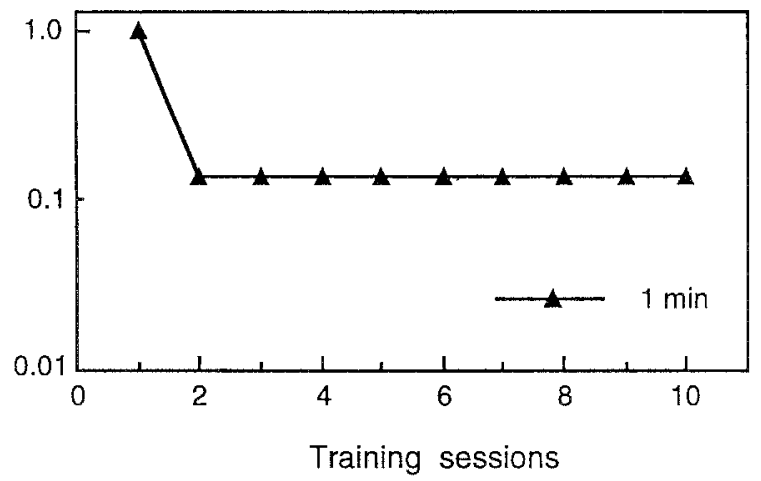

Fig. 6. Simulation of a habituation series with a $1 \mathrm{~min}$ intert-trial pause. In the simulation, the instantaneous response of the model is assumed to be proportional to the synaptic weight, $y(t)$. The log-scale is used for the ordinate. A simulated training trial stops whenever $y(t)$ falls below $y_{M}$, and $y_{M}=0.015$. Other parameters are $\tau=200 ; \alpha=3.2 ; y_{0}=1.0 ; \beta=24$.

a short-term one with relatively large $z(i, j, t)$ (see formula 8 ) and a long-term one with relatively small $z(i, j, t)$.

Can the data of the habituation series in Fig. 1 be explained by just a short-term memory (STM) model? Without a long-term memory (LTM) process (i.e., $z(i, j, t)$ never changes), $y(i, j, t)$ returns from $y_{M}$ to $\left[y_{0}-\left(y_{0}-y_{M}\right) \exp (-\alpha T / \tau)\right]$ according to (9) after a constant recovery pause $T$, where $y_{M}$ is the value of $y(i, j, t)$ at which a habituation trial is stopped. Due to a saturation phenomena, a habituation trial is stopped during experiments before an animal's response reaches 0 (Ewert and Kehl, 1978; Wang and Ewert, 1992; cf. Fig. 9). The parameter $y_{M}$ is introduced to account for this phenomena. Therefore, the synaptic weight recovers to a constant level regardless of prior habituation trials. This is illustrated in Fig. 6, which shows a simulation of one habituation series of 10 trials based on Eq. 9 with a 1 min inter-trial pause as in Fig. 1. In this simulation, $z$ is set to constant 1 . The presynaptic stimulation in (9) is also set to 1 for simplicity. As in the experimental data, each data point represents the normalized value of the overall model response over one trial. Clearly, after an initial decay, the cumulative activity during each trial is a constant, as opposed to the drastically decreasing curve during the habituation series (Fig. 1). The detailed response of the model is unimportant, since only relative response during habituation is all that matters in producing the figure. Thus, unlike in Wang and Arbib (1992), a STM model is not sufficient to simulate the data of habituation series and LTM must be taken into consideration (see also Wang, 1993).
Inhomogeneity across MP columns is embodied not only in the prey-catching activity of the first habituation trial, but also in the number of trials in a habituation series. A habituation series at nasal locations lasts longer than ones at temporal locations (Fig. 1). This can be modeled by choosing different values for $G(i)$. In the following simulations, $G(i)=0.027 \cdot 2^{\left(D_{i}-30\right) / 20}$, consistent with (7). Thus, columns corresponding to temporal locations (larger $D_{i}$ ) have larger $G(i)$ 's, so quicker transitions from STM to LTM. That is, they take a shorter time to reach profound long-term habituation. In order to model the fact that a habituation series at a location is much prolonged when preceded by habituation at a temporal location (Fig. 1A), intercolumnar interaction must change the time course of habituation in addition to shunting inhibition to $\mathrm{P} 2$ layers. The last term of (10) describes such a change by modifying $z(i, j, t)$ by the MP3 cells of columns to the left. This modification is assumed to be accomplished by presynaptic modulation on habituation synapses, shown in Fig. 3. After habituation of an MP column, reduced MP3 spontaneous activity has the following effects on columns to the right (nasal locations): it reduces the value of the last term of $(10)$, slows down the transition from STM to LTM, and thus prolongs their habituation series.

To compare with the quantitative data of Fig. 1, the above MP model was simulated. The preprocessing by retina, tectum, and anterior thalamus (Ewert's loop(2)) to a $4^{\circ} \times 20^{\circ}$ worm-like rectangle was carried out using those models developed previously (Cervantes-Pérez et al., 1985; Wang and Arbib, 1991). For each array of an MP cell type in a column, 50 cells were included ( $n=50$, see Fig. 2 B). Time was measured like this: a basic discretization step 0.05 corresponded to $1 \mathrm{sec}$. Besides intercolumnar interactions and changes described above, a single column model followed what was previously given (Wang and Arbib, 1992, see Appendix). To show intraocular influence, Fig. 7 provides the initial habituation trials of the two columns corresponding to $30^{\circ}$ and $45^{\circ}$ respectively, one before and one after a habituation series at the other visual location. In the simulation, a habitation series was obtained using the same procedure as in the experiment (Fig. 1). That is, a habituation trial was stopped as long as the model response saturated and there was 1 min inter-trial pause. The model response to the stimulus presented at the $30^{\circ}$ angle is greatly facilitated following a habituation series at the temporal locus $\left(45^{\circ}\right)$, whereas the response to the stimulus at $45^{\circ}$ is suppressed following a habituation series at the nasal locus $\left(30^{\circ}\right)$. 


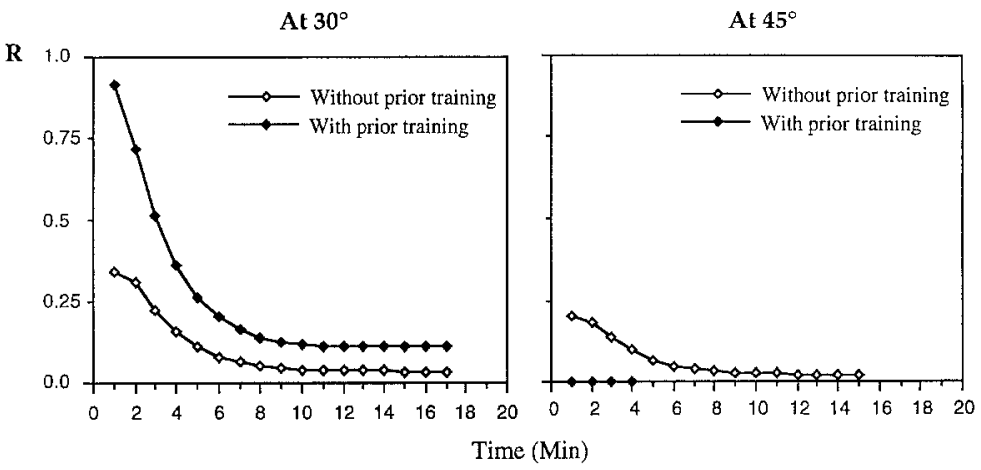

Fig. 7. Intraocular influence on habituation. The response was taken from the OUT cell of each corresponding MP column. The left frame shows two habituation trials conducted at the $30^{\circ}$ angle, one without and one with a prior habituation series done at the $45^{\circ}$ angle. The right frame show two trials conducted similarly at the $45^{\circ}$ angle. $R$ represents normalized activity of prey-catching response. The parameter values are: $B_{p 2}=0.1 ; \tau=200 ; \alpha=3.2 ; y_{0}=1.0 ; \beta=24 ; \gamma=0.1 ;$ and $z(i, j, 0)=0.9999$

Figure 8 presents the simulation result of locus specificity. As in the experiment, the five visual locations tested were $30^{\circ}, 45^{\circ}, 60^{\circ}, 90^{\circ}$, and $120^{\circ}$, corresponding to $D_{i}$ 's of (8). Computer simulations follow the same experimental conditions as in Fig. 1. In order to compare the effects of intraocular influence on habituation, Fig. $8 \mathrm{~A}$ presents the simulation results of habituation series conducted at each locus independently without any prior habituation. The figure depicts initial inhomogeneity at different visual loci. In Fig. 8B and Fig. 8C, habituation series were successively conducted from temporal to nasal locations and from nasal to temporal locations, respectively. Compared to Fig. 8A, it is clear that the model response is facilitated following prior habituation series conducted at nasal locations, but inhibited following prior habituation series at temporal locations. Furthermore, a habituation series at a particular locus was prolonged following prior habituation to the temporal direction (Fig. 8B), and the reverse was seen following prior habituation to the nasal direction (Fig. 8C). These results well match the behavioral findings of Fig. 1. The simulation results not only qualitatively reproduce locus specific habituation as shown in Fig. 1, but also exhibit close quantitative resemblance to the behavioral data in terms of the number of trials and activities in each series.

In the model, columnar interactions lead to intraocular relationships of habituation. In particular, influence of lateral connections on $z$ (see Eq. 10) results in prolonging of habituation series. We found that influence of lateral connections on other learning parameters, such as $\alpha$ and $\beta$, was unable to yield comparable results. This suggests changes in the time course of habituation series as a result of modifying long-term learning, not short-term learning. The oscillatory fluctuations of the curves in Fig. 1 A do not seem to be reliable, and may call for additional mechanisms (see Ewert, 1984).

Finally, we simulated dishabituation between two pairs of stimuli from Fig. 2A to demonstrate that this expanded MP structure with sharing of the conversion network and intercolumnar interaction is consistent with the previous single column model (Wang and Arbib, 1992) that exhibits stimulus specific habituation at a specific location. In this set, an isosceles triangle a was tested against stimuli $\mathbf{d}$ (a rectangle) and $\mathbf{b}$ (a triangle). In Fig. 9A, $\mathbf{d}$ was first presented and habituated, and a was then tested. A full new response to the later presentation was exhibited. When the order of presentation was reversed, however, no significant dishabituation occurred (Fig. 9B). If $\mathbf{b}$ was first presented and habituated, as shown in Fig. 8C, a was able to trigger a new response. But in the inverse situation (Fig. 9D) b was not able to dishabituate the habituated response to a. Figure $9 \mathrm{E}$ shows the corresponding experimental results. A comparison between Fig. 9A-D and Fig. 9E shows that the MP model clearly reproduces the experimental data.

\section{Predictions and Discussion}

The computational model of MP organization and detailed simulation of locus specificity yield a number of neurobiological predictions, as presented in the following.

(1) Organization of the medial pallium. The model pre- 
A
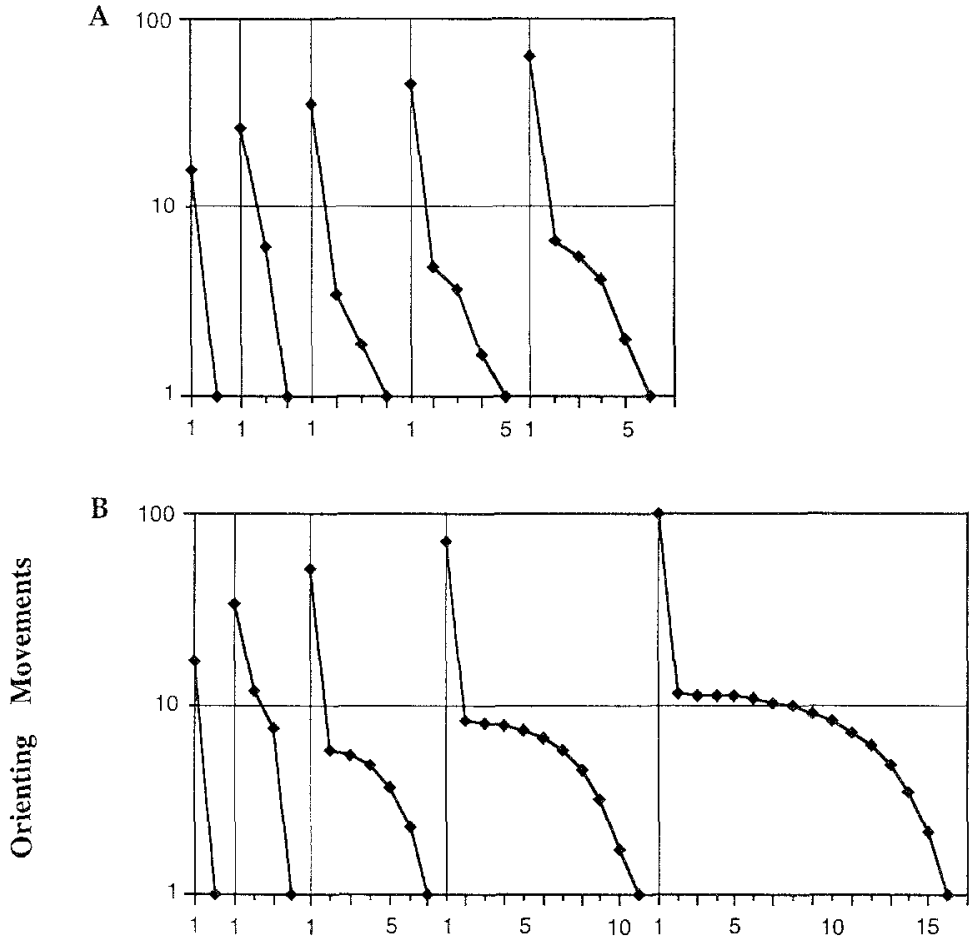

C

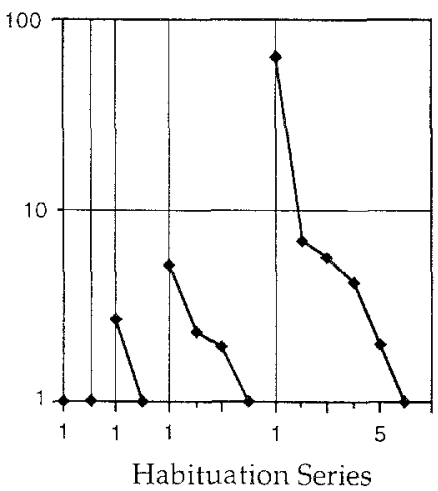

Fig. 8. Simulation of locus specific habituation. The results are plotted in the same manner as in Figure 1. To generate a dot, the overall activity is first collected by accumulating the prey-catching response (see Fig. 7) elicited by the $4^{\circ} \times 20^{\circ}$ rectange at a certain location during a habituation trial, and then scaled against the highest one which equates to the corresponding value in Fig. 1. A Habituation series conducted independently at the five locations. B Habituation series conducted successively from temporal to nasal locations. C Habituation series conducted successively from nasal to temporal locations.

dicts that there is a distinct center within MP that receives inputs from the entire set of AT ascending fibers and projects to another structure within MP. Visual processing in such a center is common to all visual locations. MP processes visual information in neuronal columns perpendicular to the telencephalic ventricle.
(2) Locus inhomogeneity. Prey-catching response and habituation properties in anurans are not homogeneous to different visual loci. In particular, as shown in Fig. 8A, the model predicts the detailed results of independent habituation series with the prey dummy $4^{\circ} \times 20^{\circ}$ rectangle conducted at $30^{\circ}, 45^{\circ}$, $60^{\circ}, 90^{\circ}, 120^{\circ}$ from the animal's midline at about 
A

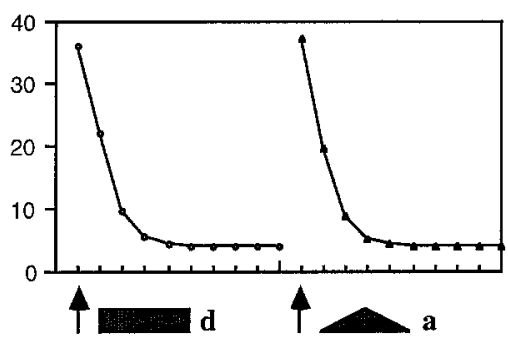

C

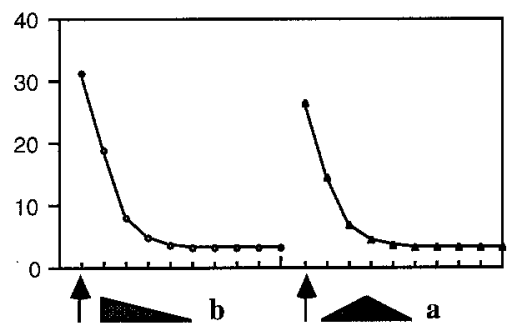

B

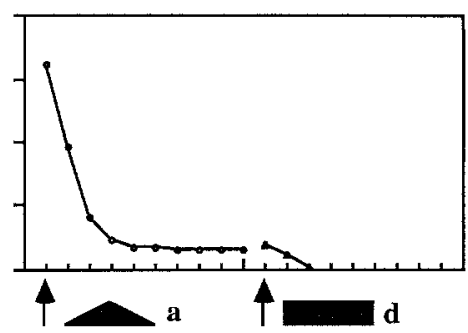

D

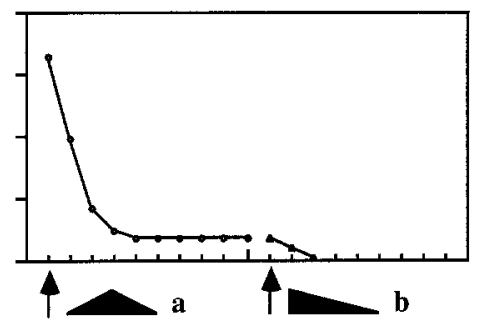

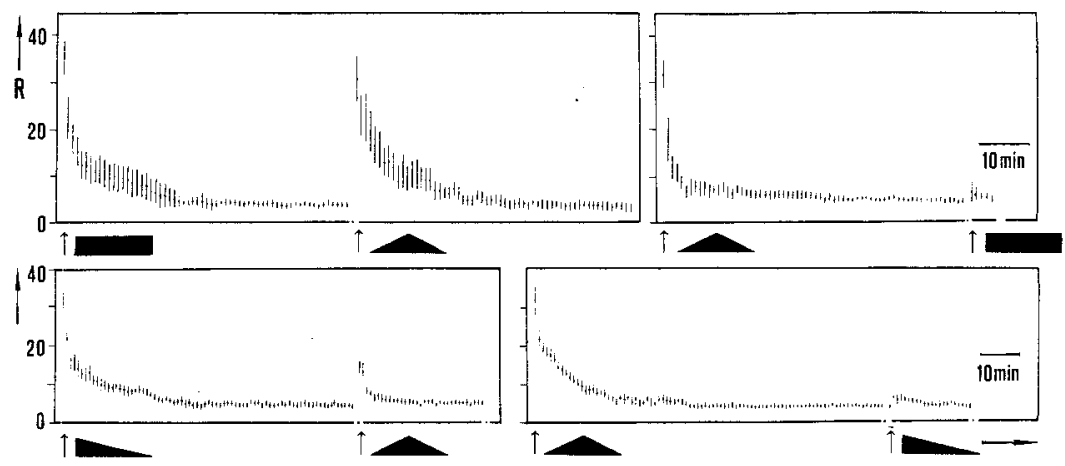

Fig. 9. Simulation of habituation and dishabituation of the orienting response in toads. In a frame, the response to the first stimulus is indicated by little circles and that to the second by little triangles. A Stimulus $\mathbf{d}$ was first presented and habituated, and then a was tested. B The reverse order of presentation as in $\mathbf{A}$. C Stimulus $\mathbf{b}$ was first presented and habituated, and then $\mathbf{a}$ was tested. D The reverse order of presentation as in C. E The corresponding experimental results by Ewert and Kehl (1978).

the same elevation as the animal's. This prediction can be tested using the same experimental procedures as in Ewert and Ingle (1971), but with negligible prior training influence (allowing long intertrial pause is one way) when a habituation series is conducted.

(3) Effects of prior training. We found from the simulation that only the immediate preceding habituation series has a significant influence on a current habituation series, because the influence built from all other earlier series has been largely diminished due to forgetting. Notice that a habituation series can last for hours, long enough to recover even moderate long-term habituation. Thus, we predict that about the same behavioral results of Fig. 1 occur even with only one preceding habituation series. That is, cumulative effects due to a number of prior series is minimal.

In this model, we propose the mechanism of sharing pattern discrimination circuitry to coordinate locus specificity and pattern discrimination. It seems to be the most parsimonious solution if an animal has the ability to discriminate many patterns at a specific location while exhibiting locus specificity. This model demonstrates that such a proposal is viable with appropriately arranged neural projections, possibly emerging from early development. The mechanism is not lim- 
ited to anurans, since pattern discrimination and locus specificity are general problems that must be solved by higher animals. It is interesting to note that there are distinct neural structures in the mammalian hippocampus: the dentate gyrus and the hippocampus proper, and the four regions CA1-CA4 of the hippocampus proper (Brown and Zador, 1990). Furthermore, longrange synaptic connections within the hippocampus are generally considered feedforward, and it has been suggested that the hippocampus plays a critical role in both pattern recognition and spatial memory (Rolls, 1988; O'Keefe and Nadel, 1978; O'Keefe, 1989). It is tempting to suggest that there are distinct regions in the hippocampus for performing pattern recognition and spatial memory, and the regions are integrated in a way similar to what is proposed here (Fig. 3).

The purpose of Sect. 3 is not to add another method of forming a topographical mapping into many already in existence, but rather to address the question of forming submaps with a single global orientation within a grand map, as required for building this MP model. Due to this requirement, putting a bias into the initial connections as suggested by von der Malsburg and Willshaw (1977) and Amari (1980) does not yield a natural solution since it would require the same ontogenetic information for each submap, quite a genetic demand. The solution proposed here, on the other hand, relies on the bias of activity gradients in the presynaptic and postsynaptic layers. The same activity gradient for each sublayer can be easily realized since a bias on the grand layer consisting of the sublayers suffices to provide all sublayers the same bias. Submaps within a global map are common phenomena in the brain. For example, in the auditory system other maps exist in iso-frequency regions of the grand tonotopic map, such as the intensity map and the spatial map (Popper and Fay, 1992).

Spontaneous activities as exhibited in MP1, MP2, and MP3 cells play an important role in the model. The decrease in MP3 activity and the increase in MP1 activity convey the after effects of long-term habituation, and these cells through their long-range projections (Fig. 3) influence other MP columns, exhibiting intraocular habituation relationships. With spontaneous activity, cells can manifest their stable activity changes, beyond just responding to an external stimulus. Additionally, cells of this type can increase or decrease their levels of activity, whereas cells without spontaneous activity can only increase their activity levels after stimulation. We suggest that spontaneous neuronal activity, as frequently observed in the central nervous system, may play the important role of manifesting long-term learning effects.

Although learning series appear to be straightforward to model, they are more intricate to simulate than single trials (see Fig. 9E), since learning series generally involve LTM processes (Wang, 1993). On the other hand, our analysis shows that conducting learning series is an effective way of obtaining quantitative LTM data, which are very valuable for elucidating computational mechanisms of LTM processes, a topic rarely treated in the literature. It is important to notice that when simulating habituation series, we did not change any parameters related to the basic model of short-term synaptic plasticity. Therefore, the present model is fully consistent with the previous model that simulates single habituation trials corresponding to short-term memory (Wang and Arbib, 1992).

Computational studies of learning in amphibians as lower vertebrates may provide valuable insights into modeling of more complex learning behaviors in higher vertebrates. Although the study reported here focuses on habituation in toads and frogs, the question thus addressed is a general one: to integrate locus specificity and pattern recognition in perception. This integration problem must be solved somehow by all higher animals. For example, on the basis of a wide variety of experiments, Sokolov $(1960,1990)$ showed habituation of the orienting response in higher vertebrates is subject to habituation, and this habituation is both locus and stimulus specific. Furthermore, he proposed a comparator theory to account for his observations, which asserts that the cortex creates a model of the stimulus that is continuously compared with the object currently presented. It was later found that cells in the rabbit hippocampus exhibit similar properties as typical behaviors in an orienting response (Vinogradova, 1975). The neural model elucidated here suggests one way of implementing the comparator model with locus specificity by a neural network. However, we realize that turning the present model into rigorous circuitry for such a task must await future research.

\section{Acknowledgments}

I thank Drs. Michael Arbib and Susan Volman for insightful discussions and critically reading the manuscript. The preparation of this paper was supported in part by NSF grant IRI-9211419 and ONR grant N00014-93-1-0335. 


\section{Appendix}

Here is the formal definition of cell types in the MP model except those defined in the text. See Wang and Arbib (1992) for a full explanation.

1. P1 Layer. The membrane potential and the output of the $j$ th $\mathrm{P} 1$ cell are

$$
\begin{aligned}
\frac{d m_{p 1}(j, t)}{d t} & =-A_{p 1} m_{p 1}(j, t)+B_{p 1} I(t)+\rho \\
N_{p 1}(j, t) & = \begin{cases}m_{p 1}(j, t) & \text { if } m_{p 1}(j, t)>\theta_{j} \\
0 & \text { otherwise }\end{cases}
\end{aligned}
$$

where $\theta_{j}=46.5 j / n+17.75$, and $I(t)$ represents input from AT. $\rho$ is the amplitude of an uncorrelated white noise introduced to the AT input.

2. MP2 Layer

$$
\begin{aligned}
\frac{d m_{m p 2}(j, t)}{d t}= & -A_{m p 2} m_{m p 2}(j, t) \\
& +\left(B_{m p 2}-m_{m p 2}(j, t)\right) I_{j j}(t) \\
& -m_{m p 2}(j, t) \sum_{k>j} I_{j k}(t) \\
I_{j k}(t)= & W_{j k} N_{p 1}(j, t) \\
W_{j k}= & \begin{cases}(k-j) / 3 & \text { if } k>j \\
1 & \text { if } k=j\end{cases} \\
N_{m p 2}(j, t)= & m_{m p 2}(j)+h_{1}
\end{aligned}
$$

3. MP3 Layer. For the $j$ th MP3 cell of column $i$

$$
\begin{aligned}
\frac{d m_{m p 3}(i, j, t)}{d t}= & -A_{m p 3} m_{m p 3}(i, j, t) \\
& +y(i, j, t) N_{m p 2}(j, t) \\
N_{m p 3}(i, j, t)= & m_{m p 3}(i, j, t)
\end{aligned}
$$

4. MP1 Layer

$$
\begin{aligned}
\frac{d m_{m p 1}(i, j, t)}{d t}= & -m_{m p 1}(i, j, t)+h_{2} \\
& -B_{m p 1} N_{m p 3}(i, j, t) \\
N_{m p 1}(i, j, t)= & \operatorname{Max}\left(m_{m p 1}(i, j, t), 0\right)
\end{aligned}
$$

5. P2 Layer. Continued from (6),

$$
C_{m p 1}=h_{2}-h_{1} y_{0} B_{m p 1}, C_{m p 3}=h_{1} y_{0} / A_{m p 3} .
$$

6. OUT Cell

$$
\begin{aligned}
\frac{d m_{\mathrm{out}}(i, t)}{d t}= & -A_{\text {out }} m_{\mathrm{out}}(t) \\
& +1 / n \sum_{j} N_{p 1}(i, j, t),
\end{aligned}
$$

and

$$
N_{\text {out }}(i, t)=m_{\text {out }}(i, t)
$$

The parameter values used in computer simulation are $A_{p 1}=1.0 ; B_{p 1}=1.0 ; \rho=0.05 ; A_{m p 2}=0.1$; $B_{m p 2}=1.1 ; h_{1}=0.6 ; A_{m p 3}=1.0 ; h_{2}=0.6 ; B_{m p 1}=$ $1.0 ; A_{\text {out }}=0.1$.

\section{References}

Amari SI (1980) Topographic organization of nerve fields. Bulletin of Mathematical Biology. 42:339-364.

Brown TH and Zador AM (1990) Hippocampus. In GM Shepherd (Ed.), The synaptic organization of the brain (pp. 346-388). New York: Oxford University Press.

Cervantes-Pérez F, Lara R, and Arbib MA (1985) A neural model of interactions subserving prey-predator discrimination and size preference in anuran amphibians. Journal of Theoretical Biology. 113:117-152

Didday RL (1976) A model of visuomotor mechanisms in the frog optic tectum. Mathematical Bioscience. 30:169-180.

Durbin R and Mitchison G (1990) A dimension reduction framework for understanding cortical maps. Nature. 343:644-647.

Eikmanns KH (1955) Verhaltensphysiologische Untersuchungen über den Beutefang und das Bewegungssehen der Erdkröte (Bufo bufo L.). Zeitschrift für Tierpsychologie. 12:229-253.

Ewert J-P (1984) Tectal mechanisms that underlie prey-catching and avoidance behaviors in toads. In $\mathrm{H}$ Vanegas (Ed.), Comparative neurology of the optic tectum (pp. 246-416). New York: Plenum.

Ewert J-P (1987) Neuroethology: toward a functional analysis of stimulus-response mediating and modulating neural circuitries. In P Ellen and C Thinus-Blonc (Eds). Congnitive processes and spatial orientation in animal and man (Pt. 1, pp. 177-200). Dordrecht. Martinus Nijhoff.

Ewert J-P, Beneke TW, Buxbaum-Conradi H, Dinges AW, Fingerling S, Glagow M, Schürg-Pfeiffer E, and Schwippert WW (1992) Adapted and adaptive properties in neural networks for visual pattern dicrimination: A neurobiological analysis toward neural engineering. Adaptive Behavior. 1:123-154.

Ewert J-P and Ingle D (1971) Excitatory effects following habituation of prey-catching activity in frogs and toads. Journal of Comparative and Physiological Psychology. 77:369-374.

Ewert J-P and Kehl W (1978) Configurational prey-selection by individual experience in the toad Bufo bufo. Journal of Comparative Physiology A. 126:105-114.

Finkenstädt T (1989a) Stimulus-specific habituation in toads: 2DG studies and lesion experiments. In J-P Ewert and M A Arbib (Eds). Visuomotor coordination: amphibians. comparisons, models, and robots (pp. 767-797). New York: Plenum.

Grossberg S (1976) Adaptive pattern classification and universal recoding: I. Parallel development and coding of neural feature detectors. Biological Cybermetics. 23:121-134.

Grossberg S and Kuperstein M (1986) Neural dynamics of adaptive sensory-motor control. Amsterdam: North-Holland.

Grossberg S and Schmajuk NA (1988) Neural dynamics of adaptive timing and temporal discrimination during associative learning. Neural Networks. 2:79-102. 
Hebb DO (1949) The organization of behavior. New York: Wiley \& Sons.

Herrick CJ (1933) The amphibian forebrain. VIII. Cerebral hemispheres and pallial primordia. Joumal of Comparative Neurology. 58:737-759.

Hoffman HH (1963) The olfactory bulb, accessory olfactory bulb and hemisphere of some anurans. Journal of Comparative Neurology. 120:317-368.

Hope RA, Hammond BJ, and Gaze RM (1976) The arrow model: retinotectal specificity and map formation in the goldfish visual system. Proceedings of Royal Society of London B. 194:447466.

Kandel ER (1976) Cellular basis of behavior: An introduction to behavioral neurobiology. New York: Freeman.

Kicliter E and Ebbesson SOE (1976) Organization of the "nonolfactory" telencephalon. In R Llinás and W Precht (Eds.), Frog Neurobiology (pp. 946-972). Berlin Heidelberg New York: Springer-Verlag.

Kohonen T (1982) Self-organized formation of topologically correct feature maps. Biological Cybernetics. 43:59-69.

Lara R and Arbib MA (1985) A model of the neural mechanisms responsible for pattern recognition and stimulus specific habituation in toads. Biological Cybernetics. 51:223-237.

Liberman MC (1978) Auditory-nerve response from cats raised in a low-noise chamber. Journal of Acoustical Society of America. 63:442-455.

Montgomery N and Fite KV (1989) Retinotopic organization of central optic projections in Rana pipiens. Journal of Comparative Neurology. 283:526-540.

Neary TJ (1990) The pallium of anuran amphibians. In EG Jones, and Peters A (Eds.) Cerebral cortex (Vol. 8A, pp. 107-138). New York: Plenum.

Northcutt RG and Kicliter E (1980) Organization of the amphibian telencephalon. In SOE Ebbesson (Ed.) Comparative neurology of the telencephalon (pp. 203-255). Plenum: New York.

Obermayer K, Ritter H, and Schulten K (1990) A principle for the formation of the spatial structure of cortical feature maps. Proceedings of National Academy of Sciences USA. 87:8345-8349.

O'Keefe J (1989) Computations the hippocampus might perform. In L Nadel, LA Cooper, P Culicover, and RM Harnish (Eds.) Neural connections, mental computation (pp. 225-284). Cambridge MA: MIT Press.

O'Keefe J and Nadel L (1978) The hippocampus as a cognitive map. Oxford: Clarendon Press.
Overton KJ and Arbib MA (1982) The extended branch-arrow model of the formation of retino-tectal connections. Biological Cybernetics. 45:157-175.

Popper AN and Fay RR (Eds. 1992) The mammalian auditory pathway: neurophysiology. New York: Springer-Verlag.

Rolls ET (1988) Visual information processing in the primate temporal lobe. In M Imbert (Ed.), Models of visual perception: from natural to artificial. Oxford: Oxford University Press.

Schiller PH (1970) The discharge characteristics of single units in the oculomotor and abducens nuclei of the unanesthetized monkey. Experimental Brain Research. 10:347-362.

Sokolov EN (1966) Neuronal models and the orienting reflex. In MAB Brazier (Ed.), The central nervous system and behavior: III (pp. 187-276). New York: Macy Foundation.

Sokolov EN (1990) The orienting response, and future directions of its development. Pavlov Journal of Biological Science. 25:142-150.

Thompson RF and Spencer WA (1966) Habituation: A model phenomenon for the study of neuronal substrates of behavior. Psychological Review. 73:16-43.

Vinogradova $O$ (1975) The hippocampus and the orienting reflex. In: EN Sokolov, and O Vinogradova (Eds.), Neuronal mechanisms of the orienting reflex (pp. 128-154). New York: Erlbaum.

von der Malsburg C (1973) Self-organization of orientation sensitive cells in the striate cortex. Kybernetik. 14:85-100.

von der Malsburg C and Willshaw DJ (1977) How to label nerve cells so that they can interconnect in an orderly fashion. Proceedings of National Academy of Sciences USA. 74:5176-5178.

Wang DL (1993) A neural model of synaptic plasticity underlying short-term and long-term habituation. Adaptive Behavior. 2:111129.

Wang DL and Arbib MA (1991) How does the toad's visual system discriminate different worm-like stimuli? Biological Cybernetics. 64:251-261.

Wang DL and Arbib MA (1992) Modeling the dishabituation hierarchy in toads: The role of the primordial hippocampus. Biological Cybernetics. 67:535-544.

Wang DL and Ewert J-P (1992) Configurational pattern discrimination responsible for dishabituation in common toads Bufo bufo (L.): Behavioral tests of the predictions of a neural model. Journal of Comparative Physiology A. 170:317-325.

Willshaw DJ and von der Malsburg C (1976) How patterned neural connections can be set up by self-organization. Proceedings of Royal Society of London B. 194:431-445. 